\title{
Perioperative Goal-Directed Therapy
}

\author{
Nathan H. Waldron, MD, Timothy E. Miller, MB, ChB, FRCA, and Tong J. Gan, MD, MHS, FRCA, Li.Ac
}

$\mathbf{M}$ ORBIDITY AFTER MODERATE-TO-MAJOR surgery remains a current issue, with perioperative organ dysfunction being responsible for a significant portion of these unfortunate events. The topic of perioperative goal-directed therapy (GDT), defined as the deliberate optimization of hemodynamics and oxygen delivery using intravenous fluid and/or vasoactive infusions, has been increasing in popularity over the last 20 years because of its proven benefit in reducing perioperative risk. This benefit likely is because of a reduction in tissue dysoxia and avoidance of organ dysfunction. Over time, perioperative GDT has been demonstrated to have both morbidity and mortality benefits for high-risk patients undergoing high-risk surgery, and there is ongoing work to define the optimal patients and situations for perioperative GDT. The authors review the history and supporting evidence behind perioperative GDT. In addition, the authors will discuss the evidence behind 2 key concepts within their current knowledge of perioperative GDT: Monitors and fluids. Finally, the authors hope to outline the status of their current knowledge regarding perioperative GDT, as well as remaining unanswered questions. The authors completed this review by searching prominent biomedical databases with the terms "perioperative" and/or "goal-directed" and "therapy", as well as "fluids", "GDT", and "hemodynamic optimization." In addition, the authors manually have searched the references of past reviews and original investigations for related papers.

\section{DEFINING PERIOPERATIVE GDT}

The primary role of anesthesiologists is to mitigate risk and improve outcomes throughout the perioperative period. While shepherding patients through the pre-, intra-, and postoperative periods, there are seemingly endless hazards to avoid and details to be checked to provide optimal care. However, despite the unique details of each and every surgical procedure, the final common pathway in many perioperative organ insults is tissue dysoxia or an imbalance between oxygen supply and demand. ${ }^{1}$ As such, an important way in which the authors are able to reduce perioperative risk and improve outcomes is through ensuring optimal end-organ perfusion during surgery.

Adverse outcomes have been associated with both underand over-resuscitation. ${ }^{2}$ Inadequate intraoperative resuscitation can lead to inadequate end-organ perfusion, ${ }^{3}$ which may worsen perioperative outcomes. ${ }^{2}$ Conversely, excessive intraoperative fluid volumes or over-resuscitation can result in increased intra- as well as extravascular volumes, which may precipitate peripheral and/or pulmonary edema. Certain surgical types, such as gastrointestinal and thoracic surgery, necessitate diligent attention to preventing perioperative fluid overload, as this may impair gastrointestinal ${ }^{2}$ and pulmonary ${ }^{4}$ function. To try to address this need for optimal tissue oxygen delivery, the concept of goal-directed therapy was introduced, whereby hemodynamic parameters and/or oxygen delivery are monitored closely (typically using flow-based monitors) and optimized with fluids and/or inotropes. ${ }^{5}$

\section{A BRIEF HISTORY OF PERIOPERATIVE GDT}

Given the remarkably high mortality and morbidity in elderly hip fracture patients in the early days, Schultz et $\mathrm{al}^{6}$ demonstrated an impressive reduction in mortality $(2.9 \% v$ $29 \%$ ) by instrumenting patients with a pulmonary artery catheter (PAC) and performing nonspecific preoperative optimization. The concept of goal-directed hemodynamic optimization began in earnest, however, with the work of Shoemaker et $\mathrm{al}^{7}$ who in 1988 showed that placement of a PAC and attainment of supraphysiologic hemodynamic parameters (ie, $\mathrm{CI} \geq 4.5 \mathrm{~L} / \mathrm{min} / \mathrm{m}^{2}, \mathrm{DO}_{2} \geq 600 \mathrm{~mL} / \mathrm{min}$ ) were associated with a greater chance of survival in high-risk surgical patients. In addition, Shoemaker et $\mathrm{al}^{8}$ conducted a prospective cohort study of 300 surgical patients with septic shock and discovered that survivors had a higher cardiac index as well as higher oxygen consumption and delivery. Drawing on this work, other investigators tested early GDT protocols and found decreased mortality in patients who received preoperative PAC and hemodynamic optimization. ${ }^{9}$ From these discoveries came the concept of superoptimization or using vasopressors and inotropes to target supranormal indices of cardiac performance and oxygen delivery. Superoptimization has been associated with mixed outcomes. Using inotropes to increase oxygen delivery (DO2) during surgery decreased perioperative morbidity and mortality in high-risk surgical patients ${ }^{9}$ as well as patients undergoing major elective surgical procedures. ${ }^{10}$ In addition,

From the Department of Anesthesiology, Duke University, Durham, North Carolina.

T.E.M. is a consultant for Edwards Lifesciences, Covidien, and Hospira. Research funding from Cheetah Medical and Retia Medical. T.J.G. is a consultant for Baxter, Edwards Life Science, Hospira and QRx. Research support from AcelRx, Cheetah, Covidien, Cubist, Deltex, Fresenius, Merck, Pacira and Premier.

This work was supported solely by departmental funds.

Address reprint requests to Tong J. Gan, MD, MHS, FRCA, Professor and Chairman, Department of Anesthesiology, Stony Brook University HSC Level 4, Rm 060 Stony Brook, NY 11794-8480 Tel: (631) 444-2979 Fax: (631) 444-2907 E-mail: tong.gan@ stonybrookmedicine.edu

(C) 2014 Elsevier Inc. All rights reserved.

1053-0770/2601-0001\$36.00/0

http://dx.doi.org/10.1053/j.jvca.2014.07.008

Key words: hemodynamic optimization, goal-directed therapy, perioperative, high risk, goal-directed fluid therapy 
patients undergoing cardiothoracic surgery, ${ }^{11}$ as well as major general surgery, ${ }^{12}$ whose oxygen delivery was superoptimized upon arrival in the intensive care unit (ICU) had decreased hospital length-of-stay (LOS). However, studies utilizing superoptimization in septic ${ }^{13}$ as well a mixed group of critically ill ICU patients ${ }^{14}$ have not demonstrated an effect on mortality. However, these studies have been critiqued for starting resuscitation $>12$ hours after arrival to the ICU, after patients perhaps had irreversible tissue dysoxia and organ damage.

Not long after the work of Shoemaker et al, Mythen et $\mathrm{al}^{15}$ demonstrated a significant relationship between gut mucosal hypoperfusion during high-risk surgery and postoperative complications, including mortality. Interestingly, it was noted that patients who did not experience gut mucosal hypoperfusion had an increase in cardiac index during surgery, whereas patients who did experience hypoperfusion did not have an intraoperative increase in CI. Mythen et al followed this with the initial intraoperative goal-directed fluid therapy study, in which patients undergoing cardiac surgery receiving intravenous colloid boluses with the goal of optimizing stroke volume and central venous pressure (CVP) were found to have a lower incidence of gastrointestinal (GI) mucosal hypoperfusion, major complications, as well as shorter hospital and ICU LOS. ${ }^{3}$ Following this work, Sinclair et al $^{16}$ utilized esophageal Doppler-guided GDT in 40 patients undergoing repair of femoral neck fractures and found that patients receiving colloid boluses to optimize stroke volume (SV) and corrected flow time had shorter hospital stays than control patients. With a growing literature base, the study and practice of GDT began to increase.

\section{ESTABLISHED BENEFITS OF GDT}

Perioperative GDT repeatedly has been associated with improved outcomes following moderate-to-major surgery, including shorter hospital LOS, fewer ICU admissions, fewer GI complications, and decreased rates of acute kidney injury. ${ }^{2,3,16-21}$ Excitingly, there are emerging data that suggest a long-term survival benefit (up to 15 years postoperatively) in ICU patients who underwent perioperative GDT associated with high-risk surgery. ${ }^{22}$ In addition, there are 2 recent metaanalyses that show mortality and morbidity benefits in patients undergoing perioperative GDT. ${ }^{23,24}$ However, a recent large multicenter prospective trial of hemodynamic optimization versus usual care in high-risk patients undergoing major gastrointestinal surgery showed no difference in postoperative morbidity or mortality, although an up-to-date meta-analysis including these data still shows a reduction in morbidity with perioperative GDT. ${ }^{25}$ A brief overview of the supporting evidence for perioperative GDT will be reviewed here.

By optimizing oxygen delivery, GDT may improve perfusion of microvascular beds in the splanchnic circulation, thus improving postoperative bowel function. Two recent metaanalyses have shown reductions in postoperative nausea and vomiting (PONV) and ileus, ${ }^{26}$ as well as a faster return of normal GI function, ${ }^{18}$ in patients receiving perioperative GDT. Of note, these reviews and others ${ }^{27}$ have found significantly fewer postoperative complications and shorter hospital LOS in GDT patients. A 2009 meta-analysis showed a reduction in both minor (eg, PONV) and major (eg, anastomotic leak) GI complications in patients receiving GDT. ${ }^{19}$ These differences may result from improved visceral perfusion as well as an avoidance of interstitial edema, as there are animal data that increased volumes of crystalloid are associated with weaker intestinal anastomoses. $^{28,29}$

Though GDT optimizes intravascular volume and oxygen delivery, concerns have been raised ${ }^{30}$ that volume expansion during GDT could result in overload and/or cardiac decompensation. Furthermore, routine exposure to vasoactive infusions to optimize oxygen delivery is not without risk. As such, Arulkumaran et $\mathrm{al}^{31}$ performed a meta-analysis of 22 trials that did not reveal any increase in cardiovascular risk in patients treated with GDT. In fact, they demonstrated a reduction in the risk of cardiovascular complications in patients receiving GDT that was most notable in studies using fluids and inotropes, supranormal oxygen delivery goals, and minimally invasive cardiac output monitors (ie, not a PAC). ${ }^{32}$

Ultimately, it would be ideal if the reduction in morbidity associated with GDT led to a reduction in perioperative mortality. In fact, 3 recent reviews demonstrated that GDT reduced perioperative mortality, potentially by reducing the number of postoperative complications. In a meta-analysis of 32 trials of perioperative GDT focusing on maintaining tissue perfusion (ie, optimizing cardiac index and/or DO2 or oxygen consumption), Gurgel and do Nascimento ${ }^{23}$ found that although GDT reduced the incidence of organ dysfunction in all patients, it reduced mortality only in cohorts in which the baseline perioperative mortality exceeded $20 \%$. In addition, a meta-analysis of 29 trials of perioperative GDT with various goals and monitoring techniques by Hamilton et $\mathrm{al}^{24}$ found reductions in morbidity and mortality in GDT patients, but did note that subgroup analyses showed a mortality benefit predominantly in older trials, trials using a PAC, trials utilizing vasoactive infusions, and those targeting supranormal values. Similarly, Poeze et $\mathrm{al}^{33}$ found decreased odds of mortality in perioperative GDT patients while noting in subgroup analyses that this benefit was found only in patients in whom supranormal values were targeted. Together, these data may suggest that perioperative GDT reduces a vast array of complications, and may reduce mortality in high-risk patient groups who receive aggressive GDT.

\section{IMPORTANT CONSIDERATIONS IN PERIOPERATIVE GDT}

\section{Monitors/Goals}

Unfortunately, there is no one best endpoint for perioperative GDT. The ideal endpoint would be representative of endorgan perfusion, readily available in the perioperative period, continuous, and reproducible. Traditional pressure-based parameters such as blood pressure (BP), heart rate (HR), CVP, and pulmonary artery occlusion pressure (PAOP or wedge pressure) are appealing as they are readily available. Unfortunately, these measures all fall short as accurate endpoints for perioperative GDT. Both HR and BP have been demonstrated to be insensitive indicators of volume status, ${ }^{26}$ and it has been proposed that an intraoperative goal of normotension is inferior to GDT. ${ }^{34}$ The utility of CVP and $\mathrm{PAOP}$ as measures of preload have been questioned in both 
healthy volunteers ${ }^{35}$ and critically ill patients, ${ }^{36}$ and some investigators have shown no benefit and increased complications in high-risk surgical patients receiving a PAC. ${ }^{37}$ However, a recent meta-analysis of perioperative GDT demonstrated a morbidity reduction regardless of the monitoring technique, but a mortality reduction only in patients monitored with PAC. ${ }^{24}$

Flow-based parameters such as SV and cardiac output (CO) increasingly are being used for perioperative GDT. Although the "gold standard" for $\mathrm{CO}$ measurement is the PAC, the rare complications associated with PAC insertion prompted the development of alternative, often less-invasive, monitors. The esophageal Doppler (EDM) is a thin probe placed in a patient's esophagus that measures descending aortic blood flow and using a nomogram to calculate aortic cross-sectional area, transforms this into $\mathrm{CO}$ and $\mathrm{SV} .{ }^{38}$ The EDM is accurate for use in GDT, ${ }^{17,39}$ where its use has been shown to be superior to usual care in terms of LOS and postoperative complications. ${ }^{16,17,20}$ Given the broad evidence base supporting the use of the EDM for GDT, it is recommended for use by the National Institute of Health and Clinical Excellence. ${ }^{40}$ Pulse contour techniques (eg, LiDCO, FloTrac/Vigileo) use the arterial waveform to calculate $\mathrm{CO}$ and $\mathrm{SV}$, and also often will calculate dynamic measures such as stroke-volume variation (SVV) and/or pulse-pressure variation (PPV). Perioperative GDT using the FloTrac/Vigileo has been shown to decrease postoperative wound infection, ${ }^{41}$ whereas LiDCO-guided GDT was associated with decreased postoperative complications and shorter hospital LOS. ${ }^{12}$ Transthoracic bioimpedance/bioreactance techniques are an emerging area of interest that have been shown to be equivalent to the EDM for perioperative GDT. ${ }^{42}$

Dynamic parameters such as SVV, PPV, and pleth variability index (PVI) are gaining popularity as endpoints for perioperative GDT. Intraoperative SVV-guided GDT decreased GI complications in patients undergoing major abdominal surgery, ${ }^{43}$ decreased wound infection in high-risk surgical patients, ${ }^{41}$ and reduced fluid volumes and nausea/vomiting in thoracic surgery patients. ${ }^{4}$ The use of PPV for intraoperative GDT has been shown to decrease length of mechanical ventilation, postoperative complications, and ICU and hospital LOS in patients undergoing high-risk surgery. ${ }^{44}$ Both SVV and PPV take advantage of cardiorespiratory interaction over a mechanically ventilated respiratory cycle and are limited by their requirement for somewhat high $(\geq 8 \mathrm{~mL} / \mathrm{kg}$ ideal body weight) tidal volumes, normal sinus rhythm, and a normal interaction between the right heart and the lungs. ${ }^{45}$ PVI and pulse oximetry plethysmographic waveform amplitude $(\Delta \mathrm{POP})$ are completely noninvasive measures that use changes in the pulse oximetry waveform to predict fluid responsiveness. ${ }^{46,47}$ Perioperative PVI-based GDT has been associated with lower intra- and postoperative lactate levels in patients undergoing major abdominal surgery. ${ }^{48,49}$

Markers of tissue well-being (lactate, $\mathrm{SvO} 2, \mathrm{SCvO} 2$, gastric tonometry) bridge the gap between hemodynamic monitoring and monitoring tissue dysoxia. Although lactate is established as a marker in septic shock, ${ }^{50,51}$ lactate has not been wellstudied as an endpoint for GDT. Similarly, $\mathrm{SvO} 2$ is not wellstudied in perioperative GDT outside of cardiac surgery, ${ }^{52}$ but perioperative GDT with $\mathrm{SCvO} 2$, a closely correlated surrogate, ${ }^{53,54}$ has been shown to reduce postoperative complications and hospital LOS in patients undergoing abdominal surgery. ${ }^{55}$ Gastric tonometry is not well-studied in perioperative GDT, but has the potential to give information about the splanchnic microcirculation, which has been linked to postoperative complications. ${ }^{3,56}$

In general, monitoring for perioperative GDT is moving toward increasingly less-invasive techniques, ${ }^{57}$ in part because of perceived complications of central venous cannulation. At this point, most all of the modern monitors, including the esophageal Doppler monitor, ${ }^{58,59}$ the pulse-contour techniques, ${ }^{60,61}$ and the transthoracic bioreactance techniques ${ }^{62,63}$ have been validated against the PAC, despite this being an imperfect "gold standard". ${ }^{64,65}$ Recently, investigators have been comparing various minimally invasive monitors to ascertain the convenience and accuracy of each method. Unfortunately, these studies often find wide variability and questionable agreement between 2 noninvasive techniques, including pulse-contour techniques and the EDM, ${ }^{31,66}$ as well as bioreactance and EDM. ${ }^{32}$ However, high levels of variability also have been found between most all minimally invasive techniques and thermodilution via PAC. ${ }^{67}$ This may have to do with imprecision of the reference technique, though, as many studies show acceptable accuracy but unacceptable limits of agreement (precision). ${ }^{68}$ Arguably more important than absolute agreement are the ability of various monitors to net similar clinical outcomes. To that end, a recent prospective trial of colorectal surgery patients demonstrated similar outcomes between patients whose perioperative GDT were titrated using a bioreactance technique (NICOM) and the EDM despite wide limits of agreement. ${ }^{42}$ Similarly, a meta-analysis of more than 2,000 patients suggested that perioperative GDT reduced morbidity regardless of the monitoring technique used, ${ }^{24}$ which has led some investigators to postulate that the benefit of GDT arises from a systematic approach to hemodynamic optimization. ${ }^{69}$

How then do the authors reconcile these findings with the assertion that patients whose supranormal oxygen delivery was targeted and whose GDT was guided by PAC had reduced mortality compared to patients with other endpoints? $?^{23,24}$ Perhaps the answer lies in the baseline risk of mortality and changes in clinical practice over time. Recent meta-analysis found that the reduction in mortality with perioperative GDT was significant only in patients with an extremely high $(>20 \%)$ mortality rate, and that mortality rate has declined significantly over time. ${ }^{24,70}$ It is likely that patients with the highest risk of mortality benefit most from aggressive GDT (ie, PAC placement, use of vasoactive substances to target supranormal hemodynamic parameters), whereas most patients derive a morbidity benefit from perioperative GDT. Unfortunately, survey data show that there is still an underutilization of perioperative GDT, partially owing to a perceived lack of benefit as well as inadequate availability of or training with various monitors. $^{71}$

One emerging area of investigation in perioperative GDT is closed-loop systems for hemodynamic optimization, whereby hemodynamic parameters (typically HR, mean arterial pressure, $\mathrm{SV}$, and a dynamic indicator of preload) are tracked continuously with software designed to meter out intravenous fluids in 
order to keep patients near the plateau portion of the Starling curve. $^{72}$ This approach has been validated against trained anesthesiologists in simulated ${ }^{72}$ as well as animal hemorrhage ${ }^{73}$ models. In addition, a prospective pilot study utilizing closedloop hemodynamic optimization for high-risk general surgery patients found the system to be successful in keeping patients in the preload-independent state (ie, $\mathrm{CI} \geq 2.5$, PPV/SVV $\leq$ $13 \%)>85 \%$ of the time. ${ }^{74}$ This burgeoning technology will require further validation, but is intriguing as a method of obtaining hemodynamic optimization while also allowing anesthesiologists to perform additional higher order tasks (eg, transfusion decisions).

\section{Fluids}

The question of which and how much intravenous fluid to use during surgery is a hotly debated topic that has been covered previously in a number of excellent reviews. ${ }^{2,75}$ GDT algorithms typically include a baseline infusion of balanced crystalloid as well as intermittent boluses of colloid to optimize $\mathrm{CO}$, sometimes in addition to vasoactive medications. The use of nonbuffered crystalloids has been associated with more metabolic derangements (hyperchloremia, metabolic acidosis) than buffered fluids, ${ }^{76}$ perhaps explaining the dominance of buffered versus nonbuffered fluids in anesthesiology. Colloids were thought to optimize the microcirculation better than crystalloids $^{77}$ and are also widely thought to have superior volume-expansion properties, allowing smaller volumes of fluid to be used with improved perioperative outcomes. ${ }^{78}$

The use of colloids in the perioperative period has recently come under scrutiny, however, as recent reviews have rebuffed the idea that colloids offer an advantage over crystalloids, $^{79}$ and meta-analyses ${ }^{80}$ as well as RCTs $^{81}$ have pointed to the increased risk of kidney injury and mortality in critically ill patients receiving hydroxyethyl starch. Although in a different patient population, this has prompted further investigation of the issue of crystalloid versus colloid in perioperative GDT. Studies comparing crystalloid-versus ${\text { colloid-based GDT in both } \text { colorectal }^{82} \text { and neurosurgical }}^{83}$ procedures have demonstrated lower IV fluid volumes in colloid-based GDT patients without any meaningful clinical differences between the 2 groups. A study comparing balanced crystalloid-versus colloid-based GDT algorithms in patients undergoing cytoreductive surgery for primary ovarian cancer demonstrated lower volumes of intravenous fluids, higher $\mathrm{CO}$, and higher $\mathrm{SV}$ in colloid patients, though they found no differences in postoperative complications of LOS. ${ }^{84}$ It appears that colloids do allow for better volume expansion, but that this is associated with little to no clinical benefit in perioperative GDT, especially when the administered volumes are modest. It may be that perioperative patients are less prone to diffuse capillary leak than septic patients, and, thus, at less risk of harm from synthetic colloids. However, this idea deserves further consideration and study before conclusions are reached.

Before the advent of GDT, there was debate as to the relative merits of "wet" 85 and "dry" 86 intraoperative fluid strategies. $^{2}$ After GDT was popularized, there have been studies showing improved outcomes primarily with increased fluid volumes with GDT. ${ }^{17,20,87,88}$ However, perioperative fluid overload has been associated with adverse outcomes, ${ }^{89,90}$ which has led investigators to compare GDT with restrictive volume regimens. Interestingly, recent studies $^{91,92}$ comparing perioperative GDT to restrictive fluid protocols in colorectal surgery found no differences in postoperative outcomes. However, these studies were performed in the context of an Enhanced Recovery After Surgery (ERAS) program, with similar fluid volumes between groups, suggesting perhaps that further study is needed to establish the role of GDT within an ERAS protocol.

\section{CONCLUSIONS AND FUTURE DIRECTIONS}

Tissue dysoxia during the perioperative period contributes to morbidity and mortality and may be mitigated by the use of perioperative GDT. The concept of perioperative GDT began initially in the 1980s after observing that patients surviving critical illness had higher indices of cardiac function and oxygen delivery and has become increasingly popular since then. At present, there exists a great deal of evidence that perioperative GDT has multiple benefits in moderate-to-major surgery, including shorter hospital LOS, fewer GI complications, fewer cardiac complications, and decreased rates of acute kidney injury. In addition, there is emerging evidence that GDT confers a perioperative mortality benefit in high-risk patients.

A number of remaining questions exist regarding the optimal conduct of perioperative GDT. At its core, optimal conduct of perioperative GDT requires both a hemodynamic goal and some form of hemodynamic monitor. ${ }^{5}$ A number of monitors (ie, PAC, EDM, pulse-contour analyses, thoracic bioreactance) have some evidence base for their use in GDT, with the EDM having the strongest evidence base currently. ${ }^{45,75}$ Further validation of other monitors versus the EDM in terms of clinical outcomes should be undertaken to demonstrate equivalence of these endpoints. In addition, further study and standardization of GDT algorithms should be performed to standardize outcomes. Moreover, it would help to better delineate which patients would benefit from fluids alone versus fluids and vasoactive medications, as well as which populations benefit from individualized optimization versus supranormal hemodynamic goals. ${ }^{93}$ Furthermore, the question of which fluids and how much during GDT remains somewhat a mystery, and will continue to benefit from further study. Finally, the burgeoning field of closed-loop hemodynamic optimization continues to mature with further investigation and may one day change the way perioperative GDT is pursued.

\section{REFERENCES}

1. Lobo SM, Rezende E, Knibel MF, et al: Early determinants of death due to multiple organ failure after noncardiac surgery in high-risk patients. Anesth Analg 112:877-883, 2011
2. Grocott MP, Mythen MG, Gan TJ: Perioperative fluid management and clinical outcomes in adults. Anesth Analg 100:1093-1106, 2005 
3. Mythen MG, Webb AR: Perioperative plasma volume expansion reduces the incidence of gut mucosal hypoperfusion during cardiac surgery. Arch Surg 130:423-429, 1995

4. Zhang J, Chen CQ, Lei XZ, et al: Goal-directed fluid optimization based on stroke volume variation and cardiac index during one-lung ventilation in patients undergoing thoracoscopy lobectomy operations: a pilot study. Clinics (Sao Paulo) 68:1065-1070, 2013

5. Bennett-Guerrero E: Hemodynamic goal-directed therapy in highrisk surgical patients. JAMA 311:2177-2178, 2014

6. Schultz RJ, Whitfield GF, LaMura JJ, et al: The role of physiologic monitoring in patients with fractures of the hip. J Trauma 25:309-316, 1985

7. Shoemaker WC, Appel PL, Kram HB, et al: Prospective trial of supranormal values of survivors as therapeutic goals in high-risk surgical patients. Chest 94:1176-1186, 1988

8. Shoemaker WC, Appel PL, Kram HB, et al: Sequence of physiologic patterns in surgical septic shock. Crit Care Med 21:1876-1889, 1993

9. Boyd O, Grounds RM, Bennett ED: A randomized clinical trial of the effect of deliberate perioperative increase of oxygen delivery on mortality in high-risk surgical patients. JAMA 270:2699-2707, 1993

10. Wilson J, Woods I, Fawcett J, et al: Reducing the risk of major elective surgery: randomised controlled trial of preoperative optimisation of oxygen delivery. BMJ 318:1099-1103, 1999

11. McKendry M, McGloin H, Saberi D, et al: Randomised controlled trial assessing the impact of a nurse delivered, flow monitored protocol for optimisation of circulatory status after cardiac surgery. BMJ 329:258, 2004

12. Pearse R, Dawson D, Fawcett J, et al: Early goal-directed therapy after major surgery reduces complications and duration of hospital stay. A randomised, controlled trial [ISRCTN38797445]. Crit Care 9: R687-R693, 2005

13. Tuchschmidt J, Fried J, Astiz M, et al: Elevation of cardiac output and oxygen delivery improves outcome in septic shock. Chest 102:216-220, 1992

14. Gattinoni L, Brazzi L, Pelosi P, et al: A trial of goal-oriented hemodynamic therapy in critically ill patients. SvO2 Collaborative Group. N Engl J Med 333:1025-1032, 1995

15. Mythen MG, Webb AR: Intra-operative gut mucosal hypoperfusion is associated with increased post-operative complications and cost. Intensive Care Med 20:99-104, 1994

16. Sinclair S, James S, Singer M: Intraoperative intravascular volume optimisation and length of hospital stay after repair of proximal femoral fracture: randomised controlled trial. BMJ 315:909-912, 1997

17. Gan TJ, Soppitt A, Maroof M, et al: Goal-directed intraoperative fluid administration reduces length of hospital stay after major surgery. Anesthesiology 97:820-826, 2002

18. Abbas SM, Hill AG: Systematic review of the literature for the use of oesophageal Doppler monitor for fluid replacement in major abdominal surgery. Anaesthesia 63:44-51, 2008

19. Giglio MT, Marucci M, Testini M, et al: Goal-directed haemodynamic therapy and gastrointestinal complications in major surgery: a meta-analysis of randomized controlled trials. $\mathrm{Br} \mathrm{J}$ Anaesth 103: 637-646, 2009

20. Venn R, Steele A, Richardson P, et al: Randomized controlled trial to investigate influence of the fluid challenge on duration of hospital stay and perioperative morbidity in patients with hip fractures. Br J Anaesth 88:65-71, 2002

21. Brienza N, Giglio MT, Marucci M, et al: Does perioperative hemodynamic optimization protect renal function in surgical patients? A meta-analytic study. Crit Care Med 37:2079-2090, 2009

22. Rhodes A, Cecconi M, Hamilton M, et al: Goal-directed therapy in high-risk surgical patients: a 15-year follow-up study. Intensive Care Med 36:1327-1332, 2010
23. Gurgel ST, do Nascimento P Jr: Maintaining tissue perfusion in high-risk surgical patients: a systematic review of randomized clinical trials. Anesth Analg 112:1384-1391, 2011

24. Hamilton MA, Cecconi M, Rhodes A: A systematic review and meta-analysis on the use of preemptive hemodynamic intervention to improve postoperative outcomes in moderate and high-risk surgical patients. Anesth Analg 112:1392-1402, 2011

25. Pearse RM, Harrison DA, MacDonald N, et al: Effect of a perioperative, cardiac output-guided hemodynamic therapy algorithm on outcomes following major gastrointestinal surgery: a randomized clinical trial and systematic review. JAMA 311:2181-2190, 2014

26. Bundgaard-Nielsen M, Holte $\mathrm{K}$, et al: Monitoring of perioperative fluid administration by individualized goal-directed therapy Acta Anaesthesiol Scand 51:331-340, 2007

27. Walsh SR, Tang T, Bass S, et al: Doppler-guided intra-operative fluid management during major abdominal surgery: systematic review and meta-analysis. Int J Clin Pract 62:466-470, 2008

28. Nessim C, Sideris L, Turcotte S, et al: The effect of fluid overload in the presence of an epidural on the strength of colonic anastomoses. J Surg Res 183:567-573, 2013

29. Marjanovic G, Villain C, Juettner E, et al: Impact of different crystalloid volume regimes on intestinal anastomotic stability. Ann Surg 249:181-185, 2009

30. Arulkumaran N, Corredor C, Hamilton MA, Ball J, Grounds RM, Rhodes A, Cecconi M: Cardiac complications associated with goal-directed therapy in high-risk surgical patients: a meta-analysis. $\mathrm{Br}$ J Anaesth 112(4):648-659, 2014

31. Davies SJ, Minhas S, Wilson RJ, et al: Comparison of stroke volume and fluid responsiveness measurements in commonly used technologies for goal-directed therapy. J Clin Anesth 25:466-474, 2013

32. Conway DH, Hussain OA, Gall I: A comparison of noninvasive bioreactance with oesophageal Doppler estimation of stroke volume during open abdominal surgery: an observational study. Eur J Anaesthesiol 30:501-508, 2013

33. Poeze M, Greve JW, Ramsay G: Meta-analysis of hemodynamic optimization: relationship to methodological quality. Crit Care 9: R771-R779, 2005

34. Bartels K, Thiele RH, Gan TJ: Rational fluid management in today's ICU practice. Crit Care 17(suppl):S6, 2013

35. Kumar A, Anel R, Bunnell E, et al: Pulmonary artery occlusion pressure and central venous pressure fail to predict ventricular filling volume, cardiac performance, or the response to volume infusion in normal subjects. Crit Care Med 32:691-699, 2004

36. Marik PE, Baram M, Vahid B: Does central venous pressure predict fluid responsiveness? A systematic review of the literature and the tale of seven mares. Chest 134:172-178, 2008

37. Sandham JD, Hull RD, Brant RF, et al: A randomized, controlled trial of the use of pulmonary-artery catheters in high-risk surgical patients. N Engl J Med 348:5-14, 2003

38. Singer M: Esophageal Doppler monitoring of aortic blood flow: beat-by-beat cardiac output monitoring. Int Anesthesiol Clin 31: 99-125, 1993

39. Roche AM, Miller TE, Gan TJ: Goal-directed fluid management with trans-oesophageal Doppler. Best Pract Res Clin Anaesthesiol 23: 327-334, 2009

40. (NICE) NIfHaCE: CardioQ-ODM oesophageal doppler monitor. NICE medical technology guidance 2011

41. Scheeren TW, Wiesenack C, Gerlach H, et al: Goal-directed intraoperative fluid therapy guided by stroke volume and its variation in high-risk surgical patients: a prospective randomized multicentre study J Clin Monit Comput 27:225-233, 2013

42. Waldron NH, Miller TE, Thacker JK, et al: A prospective comparison of a noninvasive cardiac output monitor versus esophageal 
Doppler monitor for goal-directed fluid therapy in colorectal surgery patients. Anesth Analg 118:966-975, 2014

43. Ramsingh DS, Sanghvi C, Gamboa J, et al: Outcome impact of goal directed fluid therapy during high risk abdominal surgery in low to moderate risk patients: a randomized controlled trial. J Clin Monit Comput 27:249-257, 2013

44. Lopes MR, Oliveira MA, Pereira VO, et al: Goal-directed fluid management based on pulse pressure variation monitoring during highrisk surgery: a pilot randomized controlled trial. Crit Care 11:R100, 2007

45. McGee WT, Raghunathan K: Physiologic goal-directed therapy in the perioperative period: the volume prescription for high-risk patients. J Cardiothorac Vasc Anesth 27:1079-1086, 2013

46. Sandroni C, Cavallaro F, Marano C, et al: Accuracy of plethysmographic indices as predictors of fluid responsiveness in mechanically ventilated adults: a systematic review and meta-analysis. Intensive Care Med 38:1429-1437, 2012

47. Hood JA, Wilson RJ: Pleth variability index to predict fluid responsiveness in colorectal surgery. Anesth Analg 113:1058-1063, 2011

48. Forget P, Lois F, de Kock M: Goal-directed fluid management based on the pulse oximeter-derived pleth variability index reduces lactate levels and improves fluid management. Anesth Analg 111:910-914, 2010

49. Yu Y, Dong J, Xu Z, et al: Pleth variability index-directed fluid management in abdominal surgery under combined general and epidural anesthesia. J Clin Monit Comput, 2014

50. da Silva Ramos FJ, Azevedo LC: Hemodynamic and perfusion end points for volemic resuscitation in sepsis. Shock 34(suppl): $34-39,2010$

51. Nguyen HB, Kuan WS, Batech M, et al: Outcome effectiveness of the severe sepsis resuscitation bundle with addition of lactate clearance as a bundle item: a multi-national evaluation. Crit Care 15: R229, 2011

52. Polonen P, Ruokonen E, Hippelainen M, et al: A prospective, randomized study of goal-oriented hemodynamic therapy in cardiac surgical patients. Anesth Analg 90:1052-1059, 2009

53. Dueck MH, Klimek M, Appenrodt S, et al: Trends but not individual values of central venous oxygen saturation agree with mixed venous oxygen saturation during varying hemodynamic conditions. Anesthesiology 103:249-257, 2005

54. Reinhart K, Kuhn HJ, Hartog C, et al: Continuous central venous and pulmonary artery oxygen saturation monitoring in the critically ill. Intensive Care Med 30:1572-1578, 2004

55. Donati A, Loggi S, Preiser JC, et al: Goal-directed intraoperative therapy reduces morbidity and length of hospital stay in high-risk surgical patients. Chest 132:1817-1824, 2007

56. Gutierrez G, Wulf-Gutierrez ME, Reines HD: Monitoring oxygen transport and tissue oxygenation. Curr Opin Anaesthesiol 17: 107-117, 2004

57. Funk DJ, Moretti EW, Gan TJ: Minimally invasive cardiac output monitoring in the perioperative setting. Anesth Analg 108: 887-897, 2009

58. Dark PM, Singer M: The validity of trans-esophageal Doppler ultrasonography as a measure of cardiac output in critically ill adults. Intensive Care Med 30:2060-2066, 2004

59. Madan AK, UyBarreta VV, Aliabadi-Wahle S, et al: Esophageal Doppler ultrasound monitor versus pulmonary artery catheter in the hemodynamic management of critically ill surgical patients. J Trauma 46:607-611: discussion 11-2, 1999

60. Zimmermann A, Kufner C, Hofbauer S, et al: The accuracy of the Vigileo/FloTrac continuous cardiac output monitor. J Cardiothorac Vasc Anesth 22:388-393, 2008

61. Hadian M, Kim HK, Severyn DA, et al: Cross-comparison of cardiac output trending accuracy of LiDCO, PiCCO, FloTrac and pulmonary artery catheters. Crit Care 14:R212, 2010
62. Squara P, Denjean D, Estagnasie P, et al: Noninvasive cardiac output monitoring (NICOM): a clinical validation. Intensive Care Med 33:1191-1194, 2007

63. Marque S, Cariou A, Chiche JD, Squara P: Comparison between Flotrac-Vigileo and Bioreactance, a totally noninvasive method for cardiac output monitoring. Crit Care 13:R73, 2009

64. Critchley LA, Lee A, Ho AM: A critical review of the ability of continuous cardiac output monitors to measure trends in cardiac output. Anesth Analg 111:1180-1192, 2010

65. Pugsley J, Lerner AB: Cardiac output monitoring: is there a gold standard and how do the newer technologies compare? Semin Cardiothorac Vasc Anesth 14:274-282, 2010

66. Nordstrom J, Hallsjo-Sander C, Shore R, et al: Stroke volume optimization in elective bowel surgery: a comparison between pulse power wave analysis (LiDCOrapid) and oesophageal Doppler (CardioQ). Br J Anaesth 110:374-380, 2013

67. Phan TD, Kluger R, Wan C, et al: A comparison of three minimally invasive cardiac output devices with thermodilution in elective cardiac surgery. Anaesth Intensive Care 39:1014-1021, 2011

68. Cecconi M, Rhodes A, Poloniecki J, et al: Bench-to-bedside review: the importance of the precision of the reference technique in method comparison studies-with specific reference to the measurement of cardiac output. Crit Care 13:201, 2009

69. Miller TE, Roche AM, Gan TJ: Poor adoption of hemodynamic optimization during major surgery: are The authors practicing substandard care? Anesth Analg 112:1274-1276, 2011

70. Cecconi M, Corredor C, Arulkumaran N, et al: Clinical review: Goal-directed therapy-what is the evidence in surgical patients? The effect on different risk groups. Crit Care 17:209, 2013

71. Srinivasa S, Kahokehr A, Soop M, et al: Goal-directed fluid therapy- a survey of anaesthetists in the UK, USA, Australia and New Zealand. BMC Anesthesiol 13:5, 2013

72. Rinehart J, Chung E, Canales C, Cannesson M: Intraoperative stroke volume optimization using stroke volume, arterial pressure, and heart rate: closed-loop (learning intravenous resuscitator) versus anesthesiologists. J Cardiothorac Vasc Anesth 26:933-939, 2012

73. Rinehart J, Lee C, Canales C, et al: Closed-loop fluid administration compared to anesthesiologist management for hemodynamic optimization and resuscitation during surgery: an in vivo study. Anesth Analg 117:1119-1129, 2013

74. Rinehart J, Le Manach Y, Douiri H, et al: First closed-loop goal directed fluid therapy during surgery: a pilot study. Ann Fr Anesth Reanim 33:e35-e41, 2014

75. Doherty M, Buggy DJ: Intraoperative fluids: how much is too much? Br J Anaesth 109:69-79, 2012

76. Burdett E, Dushianthan A, Bennett-Guerrero E, et al: Perioperative buffered versus non-buffered fluid administration for surgery in adults. Cochrane Database Syst Rev 12:CD004089, 2012

77. Kimberger O, Arnberger M, Brandt S, et al: Goal-directed colloid administration improves the microcirculation of healthy and perianastomotic colon. Anesthesiology 110:496-504, 2009

78. Moretti EW, Robertson KM, El-Moalem H, et al: Intraoperative colloid administration reduces postoperative nausea and vomiting and improves postoperative outcomes compared with crystalloid administration. Anesth Analg 96:611-617, 2003

79. Perel P, Roberts I, Ker K: Colloids versus crystalloids for fluid resuscitation in critically ill patients. Cochrane Database Syst Rev 2: CD000567, 2013

80. Zarychanski R, Abou-Setta AM, Turgeon AF, et al: Association of hydroxyethyl starch administration with mortality and acute kidney injury in critically ill patients requiring volume resuscitation: a systematic review and meta-analysis. JAMA 309:678-688, 2013 
81. Perner A, Haase N, Guttormsen AB, et al: Hydroxyethyl starch 130/0.42 versus Ringer's acetate in severe sepsis. N Engl J Med 367: 124-134, 2012

82. Yates DR, Davies SJ, Milner HE, Wilson RJ: Crystalloid or colloid for goal-directed fluid therapy in colorectal surgery. Br J Anaesth 112:281-289, 2014

83. Xia J, He Z, Cao X, et al: The brain relaxation and cerebral metabolism in stroke volume variation-directed fluid therapy during supratentorial tumors resection: crystalloid solution versus colloid solution. J Neurosurg Anesthesiol 2014

84. Feldheiser A, Pavlova V, Bonomo T, et al: Balanced crystalloid compared with balanced colloid solution using a goal-directed haemodynamic algorithm. Br J Anaesth 110:231-240, 2013

85. Holte K, Klarskov B, Christensen DS, et al: Liberal versus restrictive fluid administration to improve recovery after laparoscopic cholecystectomy: a randomized, double-blind study. Ann Surg 240: 892-899, 2004

86. Brandstrup B, Tonnesen H, Beier-Holgersen R, et al: Effects of intravenous fluid restriction on postoperative complications: comparison of two perioperative fluid regimens: a randomized assessor-blinded multicenter trial. Ann Surg 238:641-648, 2003

87. Wakeling HG, McFall MR, Jenkins CS, et al: Intraoperative oesophageal Doppler guided fluid management shortens postoperative hospital stay after major bowel surgery. Br J Anaesth 95:634-642, 2005

88. Conway DH, Mayall R, Abdul-Latif MS, et al: Randomised controlled trial investigating the influence of intravenous fluid titration using oesophageal Doppler monitoring during bowel surgery. Anaesthesia $57: 845-849,2002$

89. Eng OS, Goswami J, Moore D, et al: Intraoperative fluid administration is associated with perioperative outcomes in pancreaticoduodenectomy: a single center retrospective analysis. J Surg Oncol 108:242-247, 2013

90. Holte K, Sharrock NE, Kehlet H: Pathophysiology and clinical implications of perioperative fluid excess. Br J Anaesth 89:622-632, 2002

91. Srinivasa S, Taylor MH, Singh PP, et al: Randomized clinical trial of goal-directed fluid therapy within an enhanced recovery protocol for elective colectomy. Br J Surg 100:66-74, 2013

92. Brandstrup B, Svendsen PE, Rasmussen M, et al: Which goal for fluid therapy during colorectal surgery is followed by the best outcome: near-maximal stroke volume or zero fluid balance? Br J Anaesth 109: 191-199, 2012

93. Bisgaard J, Toft P: Intraoperative goal-directed fluid therapywhich goals should the authors choose? Acta Anaesthesiol Scand 58: 495-496, 2014 